\title{
Effects of Formalin Contaminated Food on Reproductive Cycle and Lifespan of Drosophila Melanogaster
}

\author{
Md. Faruque Miah", Tawhida Khanom Tania, Najmun Nahar Begum, Zobada Kanak Khan \\ Department of Genetic Engineering and Biotechnology, Shahjalal University of Science and Technology, Sylhet, Bangladesh \\ *Corresponding Author: faruque-btc@sust.edu
}

Copyright (C) 2013 Horizon Research Publishing All rights reserved.

\begin{abstract}
The toxic effects of formalin contaminated food $(0 \%$ [control], $0.1 \%, 0.25 \%, 0.5 \%$ and $1 \%$ formalin) were evaluated on the reproductive cycle and lifespan of Drosophila malanogaster. The results showed a significant descendency in the number of progeny from $F_{1}$ to $F_{5}$ generation in case of $1 \%$ and from $F_{1}$ to $F_{4}$ in $0.5 \%$ formalin. The result was found significant decreasing of the percentage of survival up to reproduction in $F_{1}$ to $F 3$ with $0.25 \%$ formalin. The ingestion of formalin in diet reduces significantly the survival percentage population in $F_{1}$ to $F_{5}$ generations. The toxic effects in survival percentage were directly related to the concentration of formalin added. Different stages of life cycle of D. malanogaster shows developmental time of life cycle was lengthened with increasing concentration of formalin in $F_{1}$ to $F_{5}$. Significant diminishing of the percentage of survival in different treatments may cause of male sterility and detrimental effects on development of $D$. malanogaster. Formalin has a significant impact on the longevity of $D$. melanogaster and the experiments showed definite correlation where more the concentration of formalin was increased, the quicker the flies died. Female showed slightly longer lifespan than male but the result was insignificant.
\end{abstract}

Keywords Formalin, Reproduction, Life Cycle, Lifespan, Drosophila melanogaster

\section{Introduction}

Formalin is a solution made up of $37 \%$ formaldehyde by weight, which is widely used as a disinfectant in many human medicines, cosmetics, antiseptic in veterinary drugs, in fungicides, textiles and embalming fluids [1]. This inexpensive chemical is now frequently used in Bangladesh as one of the most common preservatives for edible items like fish, meat, milk, fruits, etc. Recently, few media reports have brought increased attention to the problem of wholesalers and venders treating fish, meat, fruits and vegetables with formalin for long shelf life, which is harmful to human health and continuous using this contaminated food can develop respiratory and neurological problems, along with cancer, liver cirrhosis, kidney, lung, allergy, asthma and other health hazards. Continuous addition of formaldehyde through the edible items in human body may cause uncontrolled cell growth or cancer in any part of body like stomach, lung and respiratory system [2].

The fruit fly, Drosophila is belonging to the family Drosophilidae, which is recorded as rapid breeder with a lot of eggs and short life cycle. Therefore, it is being used extensively in research as a model organism [3]. The toxicity of formaldehyde is well known, but their effects on reproduction and development have received little attention. Some studies have focused on the effects of some chemical products in adults and in the different stages of development, using Drosophila as a bio indicator. Presence of formalin has been detected by the mobile courts in fruits, fish, milk, meat, etc. in Bangladesh but the concentration of formalin contamination is not entirely clear. So, D. melanogaster was used as a biological model in present research to observe the effects of different concentrations of formalin contaminated food in their reproductive cycle and life span through multiple generations and their offspring number, developmental period of life cycle and life span in multiple generations.

\section{Materials and Methods}

\subsection{Culture of Drosophila Melanogaster}

The fruit fly, D. melanogaster was collected at home and transferred to the Animal Biotechnology Laboratory of the Department of Genetic Engineering and Biotechnology (GEB) at Shahjalal University of Science and Technology (SUST), Sylhet for species identification and culture. Tomato, lemon, banana, etc. were sliced and kept on the dining table near the kitchen for fly trapping. When this slice become rotten a lot of flies were sat and flying around the rotting food. The flies were collected with drinking glass by inverting carefully and close the glass mouth by hand to avoid escaping the flies while this fly always move upward. Then the flies were transferred to the experimental bottles 
through upside down. D. melanogaster was identified by the analysis of their characteristics [4] as well as determine the sex of adult fly microscopically $[5,6]$.

D. melanogaster was cultured and maintained at room temperature on $12 \mathrm{hr}$ light $/ 12 \mathrm{hr}$ dark cycle at $70-90 \%$ relative humidity, with standardized nourishment prepared with agar, baker's yeast, cornmeal, jackfruit, water and propionic acid [7]. Unless explicitly stated this experiment was completed at an ambient room temperature (ranged between $24-28^{\circ} \mathrm{C}$ ). The cooling method was used to anesthetize the flies [8].

\subsection{Reproductive Cycle Study of Drosophila Melanogaster}

Five bottles were used and labeling with respective concentrations of formalin $(0 \%$ [control], $0.1 \%, 0.25 \%, 0.5 \%$ and $1 \%$ formalin). Drosophila were then transferred to an ice pad and put under anaesthesia. Healthy flies were then sorted by gender and separated into males and females. Six males of $D$. melanogaster were stocked with the same number of females in each bottle. The culture medium was used as substrate for feeding. Parents were removed from culture bottles after 4 days. New generation initiated its emergence after nine to twelve days from the start of the experiment $\left(\mathrm{F}_{1}\right)$. The number of progeny for each concentration was scored for both male and female after approximately two weeks from the experimental initial. The same procedure was repeated until the fifth generation through three times of observation for each concentration. The progeny of each generation was used to culture the next generation of flies to observe the effects of formalin through multiple generations. Developmental period of reproductive cycle of $D$. melanogaster in each culture bottles was observed very carefully with transferring of adult fly, egg laying, egg hatching, larvae of first, second and third instar, pupae and removal of adult fly. Observation and record were made daily and all unusual events which did not agree with the expectations were noted such as fungus occurring, death of larvae and pupae, losing of culture etc. The daily developmental time from egg-to-adult were recorded for each doses of formalin $(0 \%, 0.1 \%, 0.25 \%, 0.5 \%$ and $1 \%)$ in $\mathrm{F}_{1}$ to $\mathrm{F}_{5}$ generations.

\subsection{Procedure for Lifespan Experiment}

Ten vials were labeled accordingly with different concentrations of formalin $(0 \%, 0.1 \%, 0.25 \%, 0.5 \%$ and $1 \%$ formalin), gender of $D$. melanogaster and starting date of the experiment. Newly emerged flies from normal culture were collected and anesthetized, placed healthy 10 males and 10 females separately for each dose of formalin. Flies were maintained at $24-28^{\circ} \mathrm{C}$ on a $12: 12$ light/dark cycle with survivors transferred to fresh food vials every 5 days. This process was repeated 3 times to produce more data. $D$. melanogaster for each replication was observed over a period of 4 weeks and the number of live flies was recorded in intervals twice a week.

\subsection{Statistical Analysis}

The data were analyzed statistically using t-test. Mathematical form of t- statistic is,

$$
\mathrm{t}=\frac{\mathrm{X} 1-\mathrm{X} 2}{\mathrm{Sd} \sqrt{\frac{1}{\mathrm{~N} 1}+\frac{1}{\mathrm{~N} 2}}}
$$

Where, $\overline{\mathrm{X}}_{1}=$ Mean of treated groups/control groups, $\mathrm{X}_{2}=$ Mean of control groups/ treated groups, $\mathrm{N}_{1}=$ Total number of observation in control groups, $\mathrm{N}_{2}=$ Total number of observation in treated groups and $\mathrm{Sd}=$ Standard deviation

\section{Results}

\subsection{Effects of formalin on progeny number of Drosophila melanogaster}

The effects of formalin on progeny production was examined by culturing $D$. melanogaster with addition of different doses of formalin in culture media and finally counting their progeny through the examination of five generations. The number of average progeny obtained from three replications was observed and found diminution of number of flies in the bottles with increasing dosage of formalin (Table 1).

Table 1. Effect of formalin on mean number of progeny and their survival in percentage of Drosophila melanogaster

\begin{tabular}{|c|c|c|c|c|c|c|c|c|c|c|}
\hline \multirow{2}{*}{ Generation } & \multicolumn{10}{|c|}{ Mean progeny obtained in different treatments and survival (\%) } \\
\hline & \multicolumn{2}{|c|}{$0 \%$ (Control) } & \multicolumn{2}{|c|}{$0.1 \%$} & \multicolumn{2}{|c|}{$0.25 \%$} & \multicolumn{2}{|c|}{$0.5 \%$} & \multicolumn{2}{|c|}{$1.00 \%$} \\
\hline \multirow{2}{*}{$P_{1}$} & \multicolumn{2}{|c|}{12} & \multicolumn{2}{|c|}{12} & \multicolumn{2}{|c|}{12} & \multicolumn{2}{|c|}{12} & \multicolumn{2}{|c|}{12} \\
\hline & Progeny & Survival & Progeny & Survival & Progeny & Survival & Progeny & Survival & Progeny & Survival \\
\hline $\mathrm{F}_{1}$ & 130.67 & 100 & 124 & 94.90 & 100.33 & $76.78^{* *}$ & 95.67 & $73.21 * *$ & 67.67 & $51.79 * * *$ \\
\hline $\mathrm{F}_{2}$ & 123 & 100 & 115.67 & 94.04 & 101.67 & $82.66^{*}$ & 85.00 & $69.11 * * *$ & 62.00 & $50.40 * * *$ \\
\hline $\mathrm{F}_{3}$ & 119.33 & 100 & 124.67 & 95.47 & 103.33 & $86.59 *$ & 86.33 & $72.34 * *$ & 74.00 & $62.01 * * *$ \\
\hline $\mathrm{F}_{4}$ & 124.67 & 100 & 121.67 & 97.59 & 109.67 & 87.97 & 95.33 & $76.47 *$ & 83.67 & $67.11^{* *}$ \\
\hline $\mathrm{F}_{5}$ & 128.33 & 100 & 121.33 & 94.54 & 117.67 & 91.69 & 109.33 & 85.19 & 89.67 & $69.87 *$ \\
\hline
\end{tabular}

Significant levels of survival: ${ }^{*} \mathrm{p} \leq 0.05 ; * * \mathrm{p} \leq 0.01 ; * * \mathrm{p} \leq 0.001$ 
Differences between the ranges of survival up to maturation among the treated groups in relation to the respective control groups on each generation were observed in percentage (Table 1). In case of $0.25 \%$ formalin treated group the result was significantly decreased of the percentage of survival in $\mathrm{F}_{1}$ with values of $76.78 \%\left({ }^{* *} \mathrm{p} \leq\right.$ $0.01)$. There was significantly decreased of the survival with $82.66 \%\left({ }^{*} p \leq 0.05\right)$ in $F_{2}$ and $86.59 \%\left({ }^{*} p \leq 0.05\right)$ in $F_{3}$ generations for same dose of formalin. In $F_{4}$ and $F_{5}$ generations, survival of progeny was also decreased but the result was not significant. By $0.5 \%$ formalin use, it was found a significant diminishing of the percentage of survival with values of $73.21 \%\left({ }^{* *} \mathrm{p} \leq 0.01\right)$ in $\mathrm{F}_{1}$ until $76.47 \%\left({ }^{*} \mathrm{p} \leq\right.$ $0.05)$ in $\mathrm{F}_{4}$. The diminishing of survival was highly significant with values of $69.11 \%(* * * p \leq 0.001)$ in $F_{2}$ and $72.34 \%(* * \mathrm{p} \leq 0.01)$ in $\mathrm{F}_{3}$. But higher levels of recuperation of survival with values of $85.19 \%$ by same doses of formalin in generation $\mathrm{F}_{5}$ was observed, which was not significant with the control group. A significant diminishing of the percentage of survival in all generations of $1 \%$ treated group was also observed and values were found $51.79 \%(* * * \mathrm{p} \leq$ $0.001)$ in $\mathrm{F}_{1}$ until $69.87 \%\left({ }^{*} \mathrm{p} \leq 0.05\right)$ in $\mathrm{F}_{5}$ of same doses. In generations $F_{1}, F_{2}$ and $F_{3}$ of the same doses the diminishing of survival was highly significant which was $51.79 \%$ (*** $\mathrm{p} \leq$ $0.001), 50.40 \%\left({ }^{* * *} \mathrm{p} \leq 0.001\right)$ and $62.01 \%\left({ }^{* * *} \mathrm{p} \leq 0.001\right)$ respectfully. And, the levels of recuperation of survival was observed with values of $67.11 \%(* * p \leq 0.01)$ in $\mathrm{F}_{4}$ and 69.87 $\left({ }^{*} p \leq 0.05\right)$ in $F_{5}$. Whereas, there was no significant difference among the survivals between control group and $0.1 \%$ treated group through successive generations. The treatments of $0.1 \%$ formalin was observed a higher percentage of survival of $94.90 \%$ in $\mathrm{F}_{1}, 94.04 \%$ in $\mathrm{F}_{2}$, $104.47 \%$ in $\mathrm{F}_{3}, 97.59 \%$ in $\mathrm{F}_{4}$ and $94.54 \%$ in $\mathrm{F}_{5}$ respectively.

\subsection{Effects of Formalin on Different Stages of Reproductive Cycle of Drosophila Melanogaster}

Effects on different stages of reproductive cycle (egg-to-adult/day) of D. melanogaster were examined from $F_{1}$ to $F_{5}$ for each dosage of formalin by three replicates and compare with the respective control groups (Figure 1). The developmental time was determined through the study of reproductive cycle of $D$. melanogaster with $0.1 \%$ treated food for 7-8 days in all successive generations; however, with addition of formalin $0.25 \%, 0.5 \%$ and $1 \%$ by volume was lengthened (range 1-4 days). The main effect of the treatment found is the extreme increase in developmental time for $0.25 \%$ (until $\mathrm{F}_{2}$ ), $0.5 \%$ (until $\mathrm{F}_{3}$ ), and $1 \%$ (until $\mathrm{F}_{5}$ ).

In this experiment the percentage of formalin in media increased beyond $0.1 \%$ formalin, resulting the average of reproductive cycle of $D$. melanogaster was lengthened in each generation (Table 2). In case of $0.1 \%$ formalin use, there was no significant differences between ranges of average period of reproductive cycle of treated and control groups from $\mathrm{F}_{1}$ to $\mathrm{F}_{5}$ generations. With the treatment of $0.25 \%$ formalin in $\mathrm{F}_{1}$, the lengthening of average reproductive cycle was recorded in highly significant with values of 7.67 days $\left({ }^{* *} \mathrm{p} \leq 0.01\right)$, while $\mathrm{F}_{2}$ generation was significant $\left({ }^{*} p \leq 0.05\right)$ with values of 8.17 days when compare to the control group with values of 7.17 and 7.33 days respectfully. But in generation $\mathrm{F}_{4}$ and $\mathrm{F}_{5}$, the average reproductive cycle is longer than the respective control group but increased insignificantly (Table 2). However, significant lengthening of reproductive cycle of $D$. melanogaster of all generations with $0.5 \%$ formalin was found in $\mathrm{F}_{2}$ generation with value of 9.17 days $\left({ }^{* *} \mathrm{p} \leq 0.01\right)$ in relation to the control with values of 7.33 days, while insignificant result was recorded in $\mathrm{F}_{4}$ and $\mathrm{F}_{5}$ generations (Table 2, Figure 2). But $1.0 \%$ formalin treated group of D. melanogaster was found significant lengthening of reproductive cycle in all generations with values of 9.67 day $(* * * p \leq 0.001)$ in $F_{1}$ and 9.83 day $(* * * p \leq 0.01)$ in $F_{2}$ where 7.17 day and 7.5 day in controlled respectively, and in $F_{3}$ generation's progeny compare to the respective control group with $* * p \leq 0.01$. Moreover, in $F_{4}$ and $F_{5}$ for the same group, the average reproductive cycle was significantly increased compare to the respective control group with ${ }^{*} \mathrm{p} \leq 0.05$ (Table 2 ).

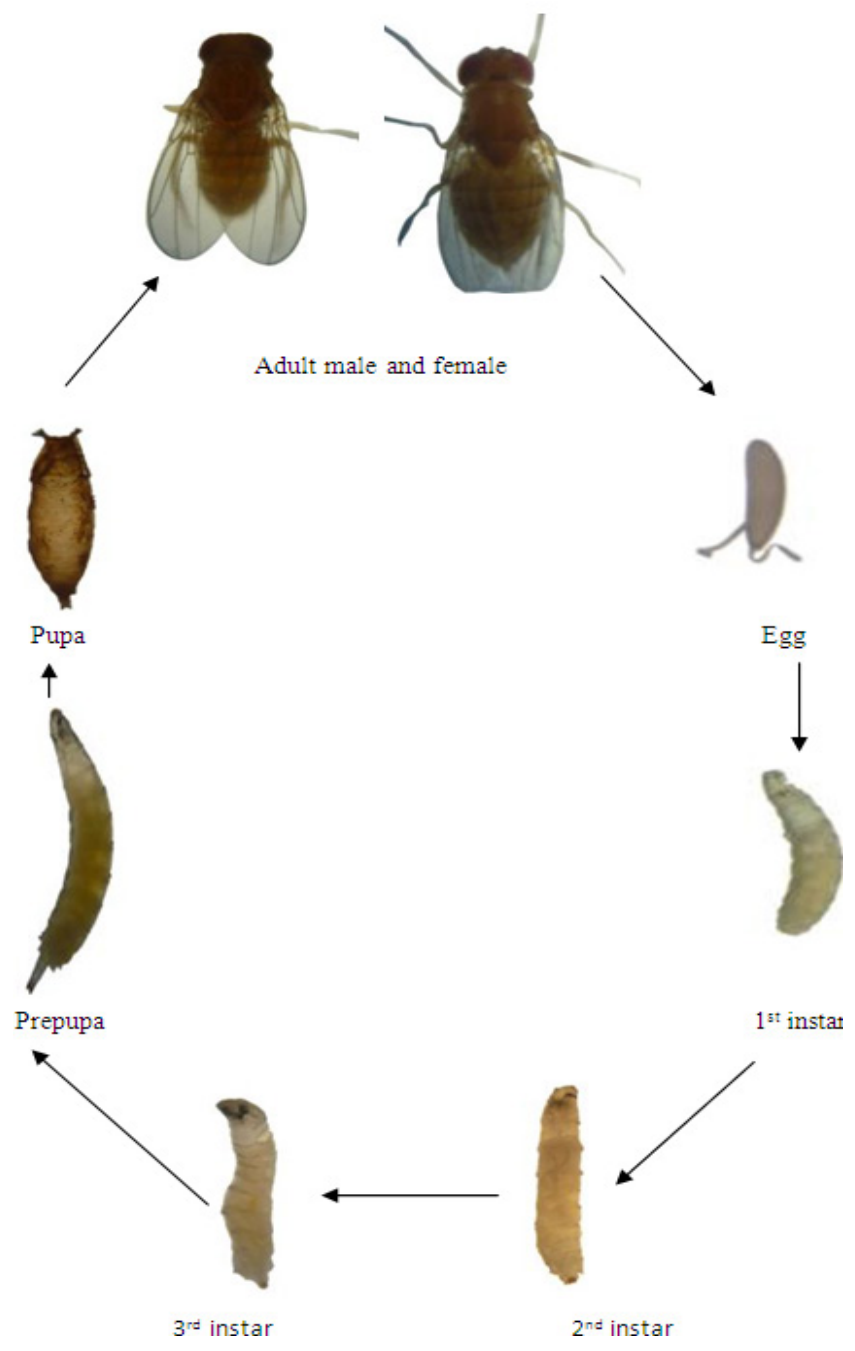

Figure 1. Observed different stages of reproductive cycle of $D$. melanogaster 
Table 2. Effects on reproductive cycle of $D$. melanogaster with different doses of formalin by three replicates

\begin{tabular}{|c|c|c|c|c|c|}
\hline & \multicolumn{5}{|c|}{ Average developmental time (in day) of life cycle of $D}$. \\
\hline Generation & $\begin{array}{c}0 \% \\
\text { (Control) }\end{array}$ & $0.1 \%$ & $0.25 \%$ & $0.5 \%$ & $1 \%$ \\
\hline $\mathrm{F}_{1}$ & 7.17 & 7.33 & $7.67^{* *}$ & $7.83^{* *}$ & $9.67^{* * *}$ \\
\hline $\mathrm{F}_{2}$ & 7.33 & 7.33 & $8.17^{*}$ & $9.17^{* * *}$ & $9.83^{* * *}$ \\
\hline $\mathrm{F}_{3}$ & 7.5 & 7.5 & 8.17 & $8.83^{*}$ & $10.17^{* *}$ \\
\hline $\mathrm{F}_{4}$ & 7.83 & 8.00 & 8.17 & 8.33 & $8.83^{*}$ \\
\hline $\mathrm{F}_{5}$ & 7.16 & 7.33 & 7.5 & 7.83 & $8.17^{*}$ \\
\hline
\end{tabular}

${ }^{*} \mathrm{p} \leq 0.05 ; * * \mathrm{p} \leq 0.01 ; * * * \mathrm{p} \leq 0.001$

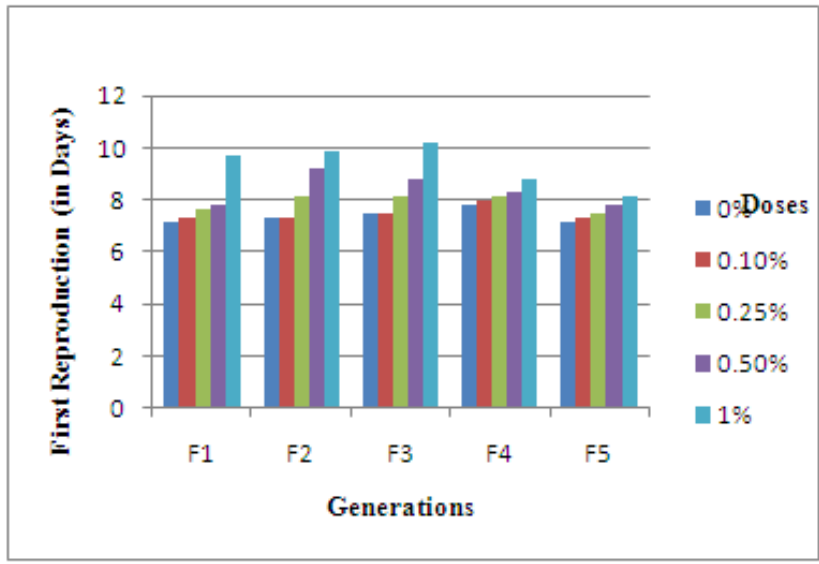

Figure 2. Graphical representation of effects on average period of reproductive cycle of $D$. melanogaster with different doses of formalin

\subsection{Formalin Effect on the Life span of Drosophila Melanogaster}

Newly emerged flies were collected from normal culture and 10 of each gender of D. melanogaster were raised separately on culture medium for life span study with addition of different doses of formalin while each treatment was replicated three times. The percentage of formalin in the media increased beyond $0.1 \%$, the number of flies alive drastically declined after a set amount of time, which was seen to be approximately four weeks (Table 3). Both $0.5 \%$ and $1.0 \%$ formalin supplementations decreased the average percentage of viability of adult flies in relation to the control group, indicating that formalin have the potential to decreased life span (Figure 3 and 4). Female showed slightly longer lifespan than male but the result was not very significant. Graphs shows a correlation between the dose level of formalin and the number of flies (male and female) alive in each bottle over time (Figure 3 and 4 ).

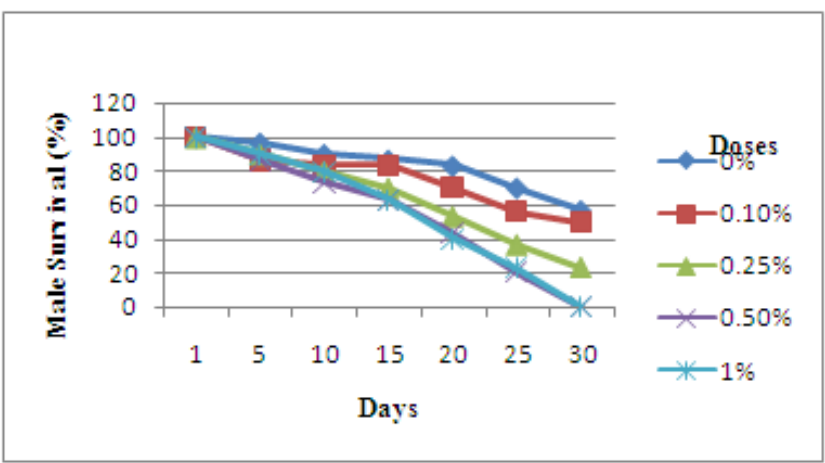

Figure 3. Lifespan curve of male D. Melanogaster

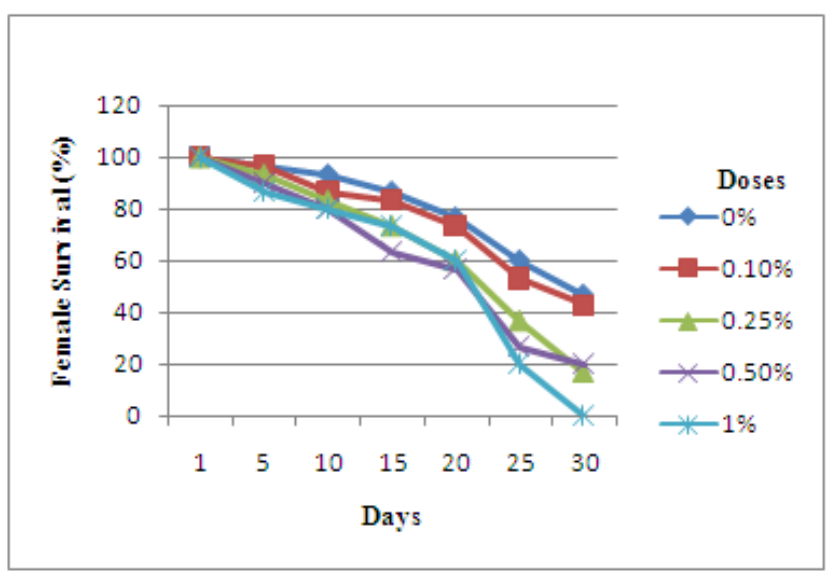

Figure 4. Lifespan curve of female D. melanogaster

Table 3. Average survival percentage of adult male and female $D$. melanogaster by different doses of formalin

\begin{tabular}{|c|c|c|c|c|c|c|c|c|c|c|}
\hline \multirow{2}{*}{$\begin{array}{c}\text { Period } \\
\text { Day }\end{array}$} & \multicolumn{5}{|c|}{ Live males } & \multicolumn{5}{|c|}{ Live females } \\
\hline & $0 \%$ (Control) & $0.1 \%$ & $0.25 \%$ & $0.5 \%$ & $1 \%$ & $0 \%$ (Control) & $0.1 \%$ & $0.25 \%$ & $0.5 \%$ & $1 \%$ \\
\hline 1 & 100 & 100 & 100 & 100 & 100 & 100 & 100 & 100 & 100 & 100 \\
\hline 5 & 96.67 & 86.67 & 90 & 86.67 & 90 & 96.67 & 96.67 & 93.33 & 90 & 86.67 \\
\hline 10 & 90 & 83.34 & 80 & 73.33 & 80 & 93.33 & 86.34 & 83.33 & 80 & 80 \\
\hline 15 & 86.67 & 83.34 & 70 & 63.33 & 63.33 & 86.67 & 83.31 & 73.33 & 63.33 & 73.33 \\
\hline 20 & 83 & 70 & 53.34 & 43.33 & 40 & 76.67 & 73.33 & 60.00 & 56.67 & 60 \\
\hline 25 & 70 & 56.67 & 36.67 & 20 & 23 & 60 & 53.33 & 36.67 & 26.67 & 20 \\
\hline 30 & 56.67 & 50.00 & 23.30 & 0.03 & 0 & 46.67 & 43.00 & 16.67 & 20 & 0 \\
\hline
\end{tabular}




\section{Discussion}

Although extensive works of formaldehyde effects on reproduction of different model organism, i.e., rat, mice, etc., are still to be done $[9,10,11]$, however, very limited study was found by the effect of formaldehyde in reproduction and life span of Drosophila. This result established that the formalin has a deep effect in the number of individuals of the descendants. The present results express an acute respond in $F_{1}$ and a chronic react in $F_{2}$ to $\mathrm{F}_{5}$ generations. Significant diminishing of the percentage of survival in case of formalin treated groups may be due to formation of male sterility in action of formaldehyde and detrimental effects on development of D. malanogaster. A research was conducted on reproductive and developmental effects of formaldehyde on $D$. melanogaster and reported the contamination of $0.25 \%$ or $0.50 \%$ of formaldehyde may inhibit the growth of larvae and adults prolong the developmental time of fly larvae and impact on their reproduction [12]. In $F_{1}$ and $F_{2}$, the lower percentage of survival in each concentration may be arisen from developmental toxicity of formaldehyde. Mutagenic effects of formaldehyde were extensively observed in male Drosophila [13], where larvae fed formaldehyde contaminated food, the primary spermatocytes were only germ cells affected and the results were found negative in case of adults, while in the present study formaldehyde contaminating medium were used from larvae to adult stage. The levels of recovery observed in $\mathrm{F}_{4}$ and $\mathrm{F}_{5}$ could have a direct relation to affect the expression of hormone "Ecdysone" of D. melanogaster regulating reproduction and development, extend the life and resistance to stress [14], and many biological effects gradually go to ordinary value at $\mathrm{F}_{4}$ generation [15]. The survival was highest among individuals from the population which had been irradiated at larval stage during many generations [16]. In case of $0.1 \%$ formalin use, no significant difference was observed in this study in relation to the control group through successive generations whereas a higher percentage of survival $94.90 \%$ in $\mathrm{F}_{1}, 94.04 \%$ in $\mathrm{F}_{2}, 104.47 \%$ in $\mathrm{F}_{3}, 97.59 \%$ in $\mathrm{F}_{4}$ and $94.54 \%$ in $\mathrm{F}_{5}$ were presented. When use sufficiently low quantities formalin to prevent bacterial growth in fish, however, growth and viability of neonates from mice given oral doses of 540 $\mathrm{mg} / \mathrm{kg}$ of body weight per day on days 8-12 of gestation were unaffected [17]. No effects on reproductive performance or on the health of offspring were observed in beagle dogs fed 0 , 3.1 , or $9.4 \mathrm{mg}$ of formaldehyde per $\mathrm{kg}$ of body weight per day in their diet on days 4-5 after mating [18]. Reproductive cycle study was performed by observing developmental time from egg-to-adult (in days) with 5 successive generations and the percentage of formalin in media increased beyond $0.1 \%$ formalin, the average reproductive cycle of $D$. melanogaster was lengthened in each generation. Toxic formaldehyde compound in formalin solution prolonged the developmental period for $D$. melanogaster. In this study effects was not examined for five stage of life cycle but it was seen through the reproductive cycle that larval stages were more sensitive to formalin and it requires more days to reach pupal stage. The developmental time in four populations of formaldehyde-treated $D$. melanogaster suggested that the effect of formalin to the culture medium of D. melanogaster was to lengthen the developmental periods [19]. This finding is in good agreement with the results of the present study. For $0.1 \%$ of formalin there was no significant difference between ranges of average reproductive cycle of treated and control group from $F_{1}$ to $F_{5}$ generation indicating that low levels of formalin in food don't extend the reproductive cycle and also don't retard growth and development.

Lifespan experiments showed that as the percentage of formalin in the media increased, the number of flies alive drastically declined after a period of time, which was seen to be approximately four weeks. Normally, the typical lifespan of D. melanogaster is approximately 30 days at $29^{\circ} \mathrm{C}\left(84^{\circ} \mathrm{F}\right)$ $[20,21], 37$ days at $25^{\circ} \mathrm{C}[22,23]$ and may be several weeks [24]. However, the majority of the flies in the control to $0.1 \%$ concentrations survived longer than that for the $0.25 \%, 0.5 \%$ and $1 \%$ concentrations (Table 3), suggesting that the flies were healthy in the control to $0.1 \%$ concentrations. After a time span of approximately 25 , the data showed that the majority of flies were dead in the concentrations of $0.5 \%$ and $1.0 \%$ treated group in compare to the control group (Figure 3 and Figure 4). Therefore, the present result is disagreed with the previous findings. The present finding suggests that the formalin must be consumed over time to have any adverse health effects, and formalin contaminated food of Drosophila is potentially toxic to D. melanogaster. The toxicity of different food additives and metal on lifespan of Drosophila were evaluated by many researcher and their results are accord with this experiment. Evaluated the toxicity of monodisperse citrate-capped gold nanoparticles (AuNPs) in D. melanogaster, upon ingestion and found a strong reduction of Drosophila lifespan [25], and the flies exposed to dosages exceeding that of $1 \%$ Ace $\mathrm{K}$ produce significantly fewer progeny and significantly shorter lifespan [26]. Indeed, longevity is partly under genetic control with mutations in single genes and stress resistance in model organisms [27]. In the present study, the number of male flies alive drastically declined but insignificant and correlation between the dosage level formalin with the percentage of flies alive for male and female respectively. The overall trend of the number of flies remaining in each vial for the lifespan experiments suggests that formalin has a significant impact on the longevity of the $D$. melanogaster.

\section{Conclusion}

The presence of formalin in the food is a growing concern due to its use in consumer food products. While toxicity of the formaldehyde is well known, but effect on reproductive cycle, development and life span have received little attention. Valuable information on the effects of formaldehyde produces was found using $D$. melanogaster. These experiments demonstrate that formalin can cause detrimental effects on reproductive and/or developmental 
success in D. melanogaster. It highlighted the importance of investigating the toxicity of formalin beyond the acute and chronic effects on D. melanogaster. These obtained results could be taken as reference about the effects of formalin in human population. The addition of formalin to commercial food products should be restrained for its detrimental effects on future generations of a variety of species.

\section{Acknowledgements}

We are very grateful to University Center of Shahjalal University of Science and Technology, Sylhet, Bangladesh for providing some funding for this study.

\section{REFERENCES}

[1] P. Feick, S. R. L. Haas, M. V. Singer, U. B. Ocker. Low-dose exposure of intestinal epithelial cells to formaldehyde results in MAP kinase activation and molecular alteration of the focal adhesion protein paxillin. Toxicology, 219: 60-72, 2006.

[2] P. F. Ross, H. Draayer, O. Itoh. An international collaborative study on a method for determination of formaldehyde in veterinary vaccines. Biologicals, 30: 37-41, 2002.

[3] C. R. Eric. Drosophila melanogaster: The fruit fly. Encyclopedia of genetics.USA: Fitzroy Dearborn Publishers, I: p157, 2001.

[4] G. Manning. A quick and simple introduction to Drosophila melanogaster. Retrieved from http://www.ceolas.org/fly/intro.html, 2006.

[5] M. Demerec, P. Kaufman. Drosophila Guide: Introduction to the genetics and cytology of Drosophila melanogaster. Cold Spring Harbor Laboratory, 4-8, 1996.

[6] D. Harry. Mendelian genetics of Drosophila. Cambridge University Press, 7-10, 2001.

[7] N. N. Begum, M. Z. Alam, A. Iqbal, M. Ashrafuzzaman, N. S. Talukder. Study on culture and life cycle of Drosophila melanogaster under various cultural and environmental conditions. International J. of Bioresearch, 10(1): 13-19, 2011.

[8] P. Geiger. Care, maintenance and manipulation of Drosophila. Retrieved

fromhttp://biology.arizona.edu/sciconn/lessons2/Geiger/intro 2.htm, 2002.

[9] E. Davidkova, K. Basmadzhieva. Changes in protein and nucleic acid metabolism as one of the methods for evaluating gonadotoxic action. Probl Khig, 4:101-109, 1979.

[10] R. Yasumura, I. Name, R. Shoji. Teratogenic and embryocidal effects of formaldehyde in mice. Teratology, 28:37A-3HA, 1983.

[11] R. Kimura, I. Naruse, R. Shoji. Embroyotoxicity of formaldehydye on developing mouse embryos. Teratology,
30: 34A, 1984.

[12] T. Abdurahman, Z. Ya, W. Yu-Feng. Effect of formaldehyde in diet on development and reproduction of Drosophila melanogaster. Journal of Environmental Entomology, 1: 12-16, 2011.

[13] C. Rohde, H. H. R. de Andrade, V. L. S. Valente. Cytogenetic analysis of two strains of Drossophila willistoni treated with formaldehyde with special references to the alcohol dehydrogenase region. Rev. Int Contam. Ambient., 12 (1): 29-34, 1996.

[14] A. Simon, C. Shih, A. Mack, S. Benzer. Steroid control of longevity in Drosophila melanogaster. Science. 299 (5611):1407-1410. 2003.

[15] J. Zhang, Q. Zhu, H. Zhao. Study of biological effects dealing with formaldehyde to Drosophila Melanogaster. Journal of Liaocheng University (Nat. Sci.), 18(2): 43-45, 2005.

[16] A. Eiche. Somatic resistance to X-ray treatment in Drosophila melanogaster populations with different irradiation backgrounds. Hereditas, 73:247-252, 1973.

[17] J. M. Seidenberg, D. G. Anderson, R. A. Becker. Validation of an in vivo developmental toxicity screen in the mouse. Teratogenesis, carcinogenesis and mutagenesis, 6:361-374, 1987.

[18] H. Hurni, H. Ohder. Reproduction study with formaldehyde and hexamethylenetetramine in beagle dogs. Food and cosmetics toxicology, 11:459-462, 1977.

[19] E. San Miguel Saln. Developmental time in four populations of formaldehyde-treated Drosophila melanogaster. Dros. Inf. Serv., 60:107, 1984.

[20] M. Ashburner, J. N. Thompson. The laboratory culture of Drosophila. In Ashburner M, Wright TRF. The genetics and biology of Drosophila, 2(A): 1-81, 1978.

[21] Ashburner, K. G. Golic, R. S. Hawley. Drosophila: A Laboratory Handbook. (2nd ed.). Cold Spring Harbor Laboratory Press. 164, 2005.

[22] L. Levine, N. M. Schwartz. Laboratory exercises in genetics (Rev Ed edition). United States, America. C. V. Mosby Company, 47-51, 1973.

[23] R. Squire. Advanced Genetics: Drosophila melanogaster culture techniques. Retrieved fromhttp://geneticslab.faywats on.com/drosophculturetech.pdf, 1-5, 2002.

[24] W. Wilson. Observing the development of Drosophila in apple juice agar. Retrieved fromhttp://www.accessescellence .org/AE/AEPC/WWC/1994/observing.php, 1994.G Vecchio, A. Galeone, B. Brunetti, G Maiorano, S. Sabella, R Cingolani, P. P. Pompa. Concentration-dependent, size-independent toxicity of citrate capped AuNPs in Drosophila melanogaster. PLoS One. 7(1): e29980, 2012.

[25] D. Huang. Effect of acesulfame potassium on phosphoinositide 3-kinase-induced viability and lifespan in Drosophila melanogaster. Acesulfame Potassium and Drosophila melanogaster, 1-26, 2011.

[26] C. J. Kenyon. The genetics of ageing. Nature, 464:504-512, 2010. 